\title{
Research on the Value and Teaching Strategies of Music Education for People with Disabilities
}

\author{
Zhice Niu, \\ ${ }^{1}$ Russian State Specialized Academy of Arts, Moscow, Russia \\ "Corresponding author. Email: niuniuntes<niuniuntes@163.com>
}

\begin{abstract}
Music is a basic course in the education system, which is beneficial to relieve emotions, repose emotions, and express thoughts, so that people can have a healthy state of mind and even gain the ability to engage in professional work. Music education for people with disabilities has certain special characteristics. Although there are lots of difficulties, the efficacy that can be obtained through music education should not be underestimated. This article will study the value and teaching strategies of music education for people with disabilities, hoping to play a positive role in promoting the teaching efficiency of special music education, promote the improvement of the overall quality of special crowd, and help them gain space for survival and development.
\end{abstract}

Keywords: People with disabilities, Music education, Educational value, Teaching strategies, Special education.

\section{INTRODUCTION}

There are approximately 90 million people with disabilities in China. This is a huge group. Today, with rapid development, this special group is facing greater pressure to survive. Quite a few of them live below the national poverty line. The issue of survival and development of them is highly valued by the country and the government, and the protection and care for them is also an important indicator to measure the degree of civilization in a society. People with disabilities have normal value realization needs. Helping them integrate into society and participate in employment is an important way for targeted poverty alleviation. Music teaching has extensive educational and therapeutic value and should be fully valued and used in special education.

\section{VALUE ANALYSIS OF MUSIC EDUCATION FOR PEOPLE WITH DISABILITIES}

People with disabilities belong to a special group, usually with physiological, psychological, and functional defects and cannot engage in certain activities in a normal way. Under normal circumstances, they have obstacles in terms of speech, vision, hearing, intelligence and physical disabilities. Music education for people with disabilities has important practical significance, which helps them to return to the mainstream life as much as possible and create value in life.

\subsection{Physiological Rehabilitation Value}

Physical health is the basis for a person's survival and development, which is particularly important for a disabled person. Music activities require the participation of all human sensory systems and limbs, which can effectively stimulate brain activity and exercise various abilities such as hearing, touch, vision, sense of space, sense of direction, and sense of balance, so that the brain can be developed, the reaction speed can be accelerated, and the body's coordination ability can be effectively improved. The survey shows that people with disabilities who have received music education have higher IQ than those who have not received music education. For some patients with cerebral palsy, music training such as clapping, nodding, stomping, and hitting can effectively improve their self-care ability, improve physical coordination, and gradually improve their limb flexibility. For some visually impaired people, although music education cannot effectively improve their vision problems, it can fully develop 
their hearing and touch, and ultimately achieve the effect of replacing vision. This also plays an important role in improving the quality of life [1].

\subsection{Psychological Rehabilitation Value}

People with disabilities have certain shortcomings themselves and lag behind ordinary people in some aspects. In the process of comparing with each other, they are prone to psychological problems, such as low self-esteem, depression, anger, hatred, and so on. Relatively speaking, the negative effects of psychological problems may be more serious than physiological problems. In today's fast-developing society, ordinary people have greater psychological burdens and competitive pressures, and people with disabilities need to have a strong heart to face the external environment and the world's attention. Since ancient times, there have been records of music treating diseases and relieving heart knots. Ouyang Xiu, a great writer in the Northern Song Dynasty, had suffered from autism and had been very depressed. Therefore, he learned to play the piano from his friend Sun Daozi, and unknowingly, his mood was relieved and his chronic disease was healed. In modern society, many people choose to listen to music to relieve their anxieties when they are upset. Instrument performance, rhythm, and changing and developing of the melody will lead people's hearts to fly freely, explore a broader space, and open the door to borderless communication, so that people with disabilities can perceive the beauty of the world in the disturbing world, sublimate their emotions, strengthen their vulnerable hearts, understand the meaning of life from a higher level, gradually eliminate psychological barriers, and return to a healthy state.

\subsection{Aesthetic Education Value}

Aesthetic level is an important aspect to measure the overall quality of an individual. In today's society, if a person has good aesthetic ability, he can get the space for survival and development, become an opinion leader in a certain field, and influence the thoughts and behaviors of the public. Therefore, it is also very important to improve the aesthetic ability, and aesthetic education has become an indispensable part of the overall development of education. People with disabilities may have to face the cruel reality. If they have the ability to recognize, appreciate, feel, experience and create beauty, then they will significantly improve their quality of life. Music is an art of expressing beauty, which can infect emotions and purify the soul. Through music education, the appreciation of excellent culture and high art is conducive to helping people with disabilities build an aesthetic system and form a correct world outlook and value. When they yearn for beauty and form an action to pursue beauty, they will be immersed in hope, thereby forgetting the defects of the body and being full of positive energy to work hard and write their own beautiful life [2].

\subsection{Quality Training Value}

To be successful, a person must not only possess basic abilities, but also possess good qualities, such as teamwork ability, emotional management ability, self-learning ability, and the ability to overcome difficulties, etc. Musical works are composed of countless notes, which require various musical instruments and different professionals to play together so as to achieve harmony and unity on the basis of their own strengths, which is conducive to cultivating cooperation and social skills. Different people with disabilities may have different emotional management problems. For example, people with intellectual disabilities may be moody, irritable and angry; people with autism may be dull, and lack of reaction and enthusiasm. And the soothing music can achieve the effect of pacifying emotions, and cheerful and rhythmic music can attract attention and awaken more emotions. People with disabilities are prone to give up when facing difficulties. There is no lack of classic works in music education that express the idea of working hard and facing difficulties. For example, in Beethoven's Symphony of Fate, from the struggle and conflict in the first chapter to the final victory and joy, it expresses his spirit of stubbornly fighting against destiny. In addition to musical works, the personal experiences of many famous musicians also have strong educational value. Beethoven completed many classic works while deaf, and the folk musician Ah Bing composed nearly 300 pieces of music in the dilemma of blindness, etc. These inspirational stories are conducive to cultivating the will and quality of people with disabilities, allowing them to find a direction to stick to and an example of learning. 


\section{RESEARCH ON THE TEACHING STRATEGIES OF MUSIC EDUCATION FOR PEOPLE WITH DISABILITIES}

\subsection{Differentiated Teaching}

Differentiated teaching is to formulate teaching strategies based on the types of deficiencies of people with disabilities, select appropriate teaching methods and organizational forms to meet their actual needs, and avoid magnification of deficiencies due to improper methods that may affect the confidence and effect of learning. For example, when facing people with intellectual disabilities, their intellectual functions and adaptability are poor. In music teaching, teachers should focus on the cultivation of cognitive ability, social skills, and psychological counseling. It is necessary for teachers to focus on teaching methods such as scene simulation, action imitation, and music game, strengthen psychological construction, establish a relationship of mutual trust with people with intellectual disabilities, and reduce the sense of fear caused by unfamiliarity. Through friendly actions such as clapping hands to say hello with the rhythm of the music, teachers can quickly establish a friendly and cooperative relationship with people with intellectual disabilities. People with intellectual disabilities have limited memory ability. Repeated imitation can stimulate their brain activity and improve memory efficiency, and teachers need to give them full confidence and patience. When facing the visually impaired, teachers should pay more attention to singing teaching and fully optimize their singing ability. Identifying the sounds of different musical instruments can make full use of their hearing to inspire imagination and open up a beautiful spiritual world. When facing autistic patients, teachers need to focus on cultivating their cognitive ability, expressive ability, and social skills. Teachers can design some teaching links such as lyrics recitation, interactive games, impromptu performances, etc., so that these patients can open their hearts in the atmosphere of music, try to communicate with others, and better adapt to the surrounding environment and interpersonal relationships. Therefore, when facing people with different types of disabilities, it is necessary for teachers to formulate targeted teaching strategies according to their characteristics, fully dig into their shining points, and meet the needs of ability improvement [3].

\subsection{Individualized Teaching}

Due to differences in living environment, growth experience, surrounding people, etc., people with the same type of disability will also show certain similarities and differences, and the difference in the degree of disability also makes the performance of various abilities different. Therefore, in addition to grasping the teaching strategy from the general direction, individualized teaching should also be implemented according to everyone's individual tendency. This is an effective supplement to differentiated teaching, putting forward higher requirements on teaching organization, teaching resources, and teacher abilities. Individualized teaching needs to be carried out on the basis of a full grasp of academic conditions, which requires teachers to fully conduct research and understand the strengths and weaknesses of different individuals. In teaching, teachers can design comprehensive activities to provide them with opportunities to demonstrate their abilities. At the same time, in the teaching process, teachers should pay attention to real-time observation of individual reactions. Some people with disabilities may change their interest points quickly, and teachers should maintain a certain degree of patience and guide them in terms of new interest points, so that they can find their own strengths driven by interest and gradually develop self-confidence. This will also drive the learning of other music projects. In addition to the organization and guidance of teachers, individuals should also be given appropriate opportunities to choose their own roles and positioning in teaching activities, which can achieve the effect of getting twofold results with half the effort [4].

\subsection{Permeable Teaching}

Relatively speaking, the teaching coordination of people with disabilities may not be as good as ordinary people. Especially for patients with intellectual disabilities, their thoughts and behaviors are more stubborn during mood swing, and the use of compulsory teaching methods may have counterproductive effects, and as a result, the real classroom efficiency may not be very good. This requires teachers to make full use of the informal teaching time to subtly integrate music into their daily lives. Music is an art form that can express emotions and repose emotions. With the help of diversified media communication channels, many popular songs can easily leave a deep impression in people's minds and make people hum 
unknowingly. This exactly is the principle of permeable teaching. Teachers should make full use of the free time after class, carefully select some music that meets the teaching goals and meet the curriculum requirements, and play it continuously during the break time, so as to assist in the rest after class, relax in the atmosphere of music, and infiltrate the content that needs to be learned in advance. Carrying out classroom teaching on this basis can make it easier for people with disabilities to quickly enter the learning rhythm. Music education doesn't have strong seriousness. It is necessary to learn to enjoy the happiness and growth brought by music. For people with disabilities, music education is more of auxiliary therapy, so it is easier to carry out permeable teaching than other courses [5].

\subsection{Integrated Teaching}

Many people with disabilities have weak abstract thinking and poor imagination. Teachers need to express more abstract things in intuitive images. With the support of multimedia technology, teachers can use a variety of expressions to teach and integrate music into specific situations and pictures, which is more conducive to creating an atmosphere, expressing thoughts and feelings, giving more sensory stimulation, and guiding people with disabilities to understand and express music in appropriate situations, and mobilize their physical and emotional enthusiasm to the greatest extent. Integrated teaching requires schools to provide support for infrastructure and educational resources, and teachers should also have strong design and application capabilities, fully demonstrate the harmony and beauty of multiple expressions, avoid the disorder and boring repetition caused by simple superposition, and be close to the life experience of the educated. In addition to the combination of teaching tools, the interaction between different courses is also very important. Music teaching can be combined with dance teaching. People with disabilities can sing and dance in rhythmic music, which not only delights the spirit, but also exercises the body, and unconsciously maintains physical and mental health [6].

\subsection{Growing Teaching}

Education should be a continuous ascent process, which can improve various abilities and comprehensive qualities on the existing basis, so that the educated can achieve phased results. Music education for people with disabilities should also adhere to the basic principle of a gradual way. According to the actual situation, teachers should formulate appropriate teaching goals and be close to the zone of proximal development of people with disabilities, so as to make sure that they can enter a higher stage of learning after completion. This not only helps to control the teaching progress, but also facilitates the systematic learning from simple to complex, making the knowledge structure system more logical. Relatively speaking, the speed of music teaching for people with disabilities should be appropriately slowed down, the pace of progress should be small, the number of cycles should be more, and it should be able to achieve multiple consolidation and reviews. On the basis of reasonably determining the teaching objectives, teachers need to do a good job of testing results, scientifically evaluate the real learning effects in a relatively relaxed environment, and create performance opportunities for people with disabilities to gain a sense of accomplishment, continue to accumulate confidence in learning, work steadily, and climb up in a thoroughgoing manner [7].

\section{CONCLUSION}

In summary, this article first elaborates the value of music education for people with disabilities, which mainly includes physiological rehabilitation value, psychological rehabilitation value, aesthetic education value, and quality training value. Then it focuses on the analysis of differentiated teaching, individualized teaching, permeable teaching, integrated teaching, and growing teaching and studies effective teaching strategies. It is hoped that it will have a positive practical significance to allow the public to fully understand the importance of music education for people with disabilities, namely, according to different types of defects, teachers should formulate effective teaching methods, respect the dominant role of the educated, give full play to the healing effect of the art of music, and help people with disabilities to grow, accumulate confidence, and find a new field of their own.

\section{AUTHORS' CONTRIBUTIONS} Niu.

This paper is independently completed by Zhice 


\section{REFERENCES}

[1] Liang Qingdong, Liang Qiuyue. The Research on the Application of Special Children's Music Education Based on Music Therapy Concept [J]. Journal of Jiangsu University of Technology, 2018,24(06):110-114. (in Chinese)

[2] Zhao Zhaling. A Review and Breakthrough of Music Education for Children with Hearing Impairment in the Context of Deaf Culture [J]. Sichuan Drama, 2019(07):161-164. (in Chinese)

[3] Sun Yun. The Inspiration of Ancient Music Education for the Disabled to Modern Special Music Education [J]. Home Drama, 2021(05):101-102. (in Chinese)

[4] Zong Lijia. The Role of Music's Aesthetic Function on the Growth of Special School Students [J]. Home Drama, 2021(06):57-58. (in Chinese)

[5] Hu Shuyi. Research and Demonstration of Using Music Therapy to Improve and Enhance the Social Adaptation of Children with Disabilities [J]. Music Space, 2016(06):32-33. (in Chinese)

[6] Du Qingqing. Discussing the Treatment Objects, Methods and Goals of Music Therapy from the Current Situation of Music Therapy for the Disabled [J]. People's Music, 2015(11): 87-89. (in Chinese)

[7] Yu Xiao, Guo Qin. The Connotation, Value and Practical Dilemma of Music Education for Special Children [J]. Journal of Suihua University, 2018, 38(04): 142-144. (in Chinese) 